\title{
High flow EC-IC bypass for a giant left MCA aneurysm
}

Eric J. Arias, M.D.1 and Gregory J. Zipfel, M.D.,2

Departments of Neurological Surgery ${ }^{1}$ and Neurology, ${ }^{2}$ Washington University School of Medicine, St. Louis, Missouri

Giant cerebral aneurysms may be treated through a variety of options, including aneurysm trapping with concurrent bypass. This video describes the case of a large, recurrent, left middle cerebral artery aneurysm that was treated using a high flow, radial artery bypass graft, from the external carotid artery to the left temporal M2 branch. A step-by-step operative description, with emphasis on proper microsurgical technique, is included.

The video can be found here: http://youtu.be/9xTMC6InivQ.

KEY WORDS EC-IC bypass; MCA aneurysm; radial artery graft; high flow bypass; aneurysm trapping 